\title{
The Application of Minimally Invasive Devices with Nanostructured Surface Functionalization: Antisticking Behavior on Devices and Liver Tissue Interface in Rat
}

\author{
Li-Hsiang Lin, ${ }^{1,2}$ Ya-Ju Hsu, ${ }^{3}$ Hsi-Jen Chiang, ${ }^{1,2,4}$ Han-Yi Cheng, ${ }^{1,4,5}$ \\ Che-Shun Wang, ${ }^{1,2}$ and Keng-Liang $\mathrm{Ou}{ }^{1,4,5,6}$ \\ ${ }^{1}$ Research Center for Biomedical Devices and Prototyping Production, Taipei Medical University, Taipei 110, Taiwan \\ ${ }^{2}$ School of Dentistry, College of Oral Medicine, Taipei Medical University, Taipei 110, Taiwan \\ ${ }^{3}$ Department of Dentistry, Sijhih Cathay General Hospital, Taipei 221, Taiwan \\ ${ }^{4}$ Graduate Institute of Biomedical Materials and Tissue Engineering, Taipei Medical University, Taipei 110, Taiwan \\ ${ }^{5}$ Research Center for Biomedical Implants and Microsurgery Devices, Taipei Medical University, Taipei 110, Taiwan \\ ${ }^{6}$ Department of Dentistry, Taipei Medical University-Shuang Ho Hospital, Taipei 235, Taiwan
}

Correspondence should be addressed to Keng-Liang Ou; klou@tmu.edu.tw

Received 28 July 2014; Revised 5 March 2015; Accepted 6 March 2015

Academic Editor: Xingcai Wu

Copyright (C) 2015 Li-Hsiang Lin et al. This is an open access article distributed under the Creative Commons Attribution License, which permits unrestricted use, distribution, and reproduction in any medium, provided the original work is properly cited.

\begin{abstract}
This study investigated the thermal injury and adhesion property of a novel electrosurgery of liver using copper-doped diamondlike carbon (DLC-Cu) surface treatment. It is necessary to reduce the thermal damage of surrounding tissues for clinical electrosurgeries. The surface morphologies of stainless steel (SS) coated with DLC (DLC-Cu-SS) films were characterized by scanning electron microscopy (SEM) and transmission electron microscopy (TEM). Bionic liver models were reconstructed using magnetic resonance imaging (MRI) to simulate electrosurgery. Cell cytotoxicity assays showed that the DLC-Cu thin film was nontoxic. The temperature of tissue decreased significantly with use of the electrosurgical device with nanostructured DLC-Cu films and increased with increasing thickness of the films. Thermography revealed that the surgical temperature in the DLC$\mathrm{Cu}$-SS electrosurgical device was significantly lower than that in the untreated device in the animal model. Moreover, compared to the SS electrosurgical device, the DLC-Cu-SS electrosurgical device caused a relatively small injury area and lateral thermal effect. The results indicate that the DLC-Cu-SS electrosurgical device decreases excessive thermal injury and ensures homogeneous temperature transformation in the tissues.
\end{abstract}

\section{Introduction}

In the last two decades, growing importance has been placed on research on electrosurgical devices in clinical excision surgery. Several studies have noted the advantages of electrosurgical devices; however, these devices cause thermal injury to the surrounding tissue $[1,2]$. Many researchers have tried to find a method to reduce this thermal damage, which can affect nearby intestines, vessels, nerves, and bile ducts [3]. So far, there are three types of electrodes used in electrosurgery, that is, blade, needle, and ball electrodes. The blade-electrode electrosurgical device using copperdoped diamond-like carbon (DLC-Cu) surface treatment was obtained from Hung-Chun Bio-S Co., Ltd., and is shown in Figure 1(a). DLC was chosen for this study due to its low electrical resistivity [4], as a result of which it would also have lower thermal effect on the electrosurgical device. Mikami et al. hypothesized that surface treatment would enhance the efficacy of radiofrequency-ablation devices, not only on the lesion but also on the adherence of the boundary tissue [5]. The adherence to bipolar forceps was dependent on both the material in the tips and its roughness. In their research, goldplated bipolar forceps demonstrated the best performance. On the other hand, a surface treatment method was used to generate a coating film to decrease the temperature of the surrounding tissue. Further other studies would focus on 


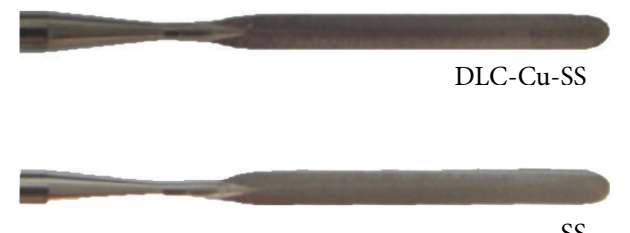

(a)

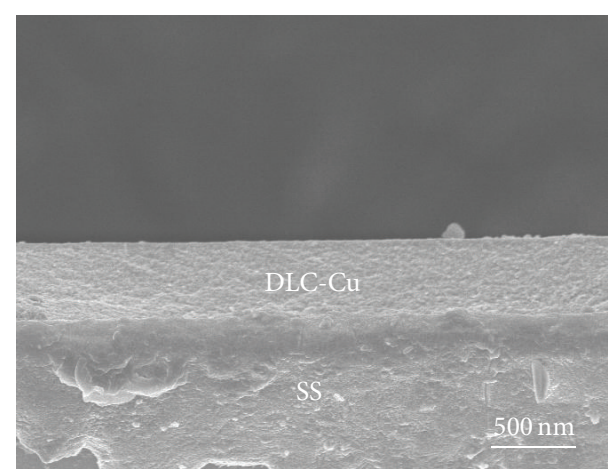

(b)

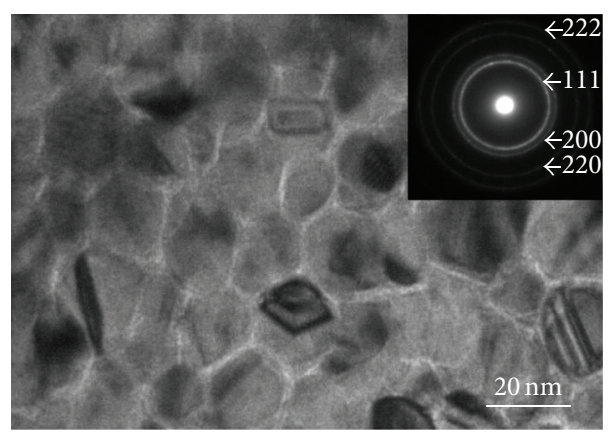

(c)

FIGURE 1: (a) Blade electrosurgical device with diamond-like carbon (DLC) surface treatment and untreated one provided from Hung-Chun Bio-S Co., Ltd., (b) SEM cross section, and (c) TEM images of the DLC-Cu-SS film.

DLC, which is capable of antiadherence. The decreased thermal injury of the tissues and platelet adherence was observed with DLC thin films and not with glass [6]. In particular, Chan et al. found that the DLC-Cu film has outstanding antibacterial properties against Escherichia coli [7]. Therefore, stainless steel (SS) coated with DLC (DLC-Cu-SS) films was proposed to coat the electrosurgical device using surface treatment technology. In the present study, the characteristics of device coated with DLC-Cu films are compared to those of the standard SS-coated electrosurgical device.

The finite element method (FEM) is useful for investigating the temperature distribution in liver and adjacent tissues. FEM has also been used previously to study the biomechanical behavior in various parts, such as hips, knees, and spinal implants, under different loading conditions [8-10]. In addition, three-dimensional (3D) finite element analysis (FEA) has been widely applied in the thermal analysis of electrosurgery [11] to evaluate the possible thermal damage to a tissue before clinical operation. Therefore, FEM is used for analyzing and comparing thermal diffusion and tissue damage caused by different surface treatments in this study.

The mechanical behavior of the electrosurgery-tissue interface is an important factor for decreasing thermal damage; however, there are few investigations of this behavior through experimental and theoretical analysis. To examine the thermal damage due to electrosurgery, the highest temperature and the dimensions of the damage region during clinical surgery should be considered. Therefore, the aim of this study is to obtain the highest temperature and calculate the damaged area in the liver by using electrosurgery with DLC-Cu-SS films.

\section{Materials and Methods}

The DLC-Cu-SS thin film was coated in a radiofrequency magnetron sputtering device. The glow discharge plasma was obtained by applying a radiofrequency input power of $225 \mathrm{~W}$ to a copper sputtering target to dope copper and synthesize the DLC thin films simultaneously. The microstructures of the DLC-Cu-SS films were characterized by using a scanning electron microscope (SEM, JEOL JSM-6500F) and a high-resolution transmission electron microscope (HRTEM, PHILIPS F-20).

Several 3D finite element models of a liver structure were generated using magnetic resonance imaging (MRI; Signa HDx 3.0T, USA). These models were reconstructed for an adult person and geometry acquisition. Many continuous images were obtained and segmented for data extraction to describe the contours of the liver. Then, an edge detection program was run with AVIZO 7.2 (Internet Securities, Inc.) to detect the various boundaries of the liver. The biomechanical properties of the liver tissue, $316 \mathrm{~L} \mathrm{SS}$, and DLC-Cu-SS films have been described in previous literatures [12-15]. The thicknesses of the treated films were 0 (SS), 100 (DLC-CuSS-1), 200 (DLC-Cu-SS-2), 300 (DLC-Cu-SS-3), 400 (DLC$\mathrm{Cu}-\mathrm{SS}-4$ ), and 500 (DLC-Cu-SS-5) nm. Then, 3D image 
reconstruction models were built using ANSYS Workbench 12.1 (ANSYS, Inc.). The mesh process should be reinforced after it is converged for more reliable results. The average numbers of nodes and elements were approximately 65,000 and 30,000, respectively. The thicknesses of the coated films varied from 0 to $500 \mathrm{~nm}$.

2.1. Cell Cytotoxicity Assay. To evaluate the cell cytotoxicity of the DLC-Cu-SS films, the samples $(n=5)$ were cultured with osteoblast cells (MG-63, ATCC CRL-1427). The variations in cell morphology following various incubation periods also were observed. Before the cells were cultured, the samples were washed in an ultrasonic bath with acetone and ethanol in succession for $15 \mathrm{~min}$ and then air-dried. Then, the samples were sterilized at room temperature for $24 \mathrm{~h}$ by using ultraviolet radiation and placed in a 24 -well polystyrene plate. Subsequently, the MG-63 cell suspensions, with a concentration of $1 \times 10^{4}$ cells $/ \mathrm{mL}$, were added to the plate and cultured in an incubator at $37^{\circ} \mathrm{C}$ with $5 \% \mathrm{CO}_{2}$ for 0,24 , and $72 \mathrm{~h}$. The extent of adhesion and proliferation of the MG-63 cells on nanostructured surfaces also was evaluated using SEM. All the cultured samples were fixed in $2 \%$ glutaraldehyde at room temperature for $1 \mathrm{~h}$. Subsequently, they were washed in a rinse buffer ( $1 \%$ phosphate buffer saline (PBS), GIBCO pH 7.4) twice and air-dried. Before being loaded into the chamber, the samples were sputtercoated with approximately $20-30 \mathrm{~nm}$ thick platinum thin film to enhance their electrical conductivity and prevent sample charging effects during SEM.

2.2. Animal Study. The protocols for the animal models were reviewed and approved by the Institutional Animal Care and Use Committee of Taipei Medical University (LAC-990037). Twelve Sprague-Dawley rats (200-300 g, BioLASCO, Taiwan) were used in accordance with the guidelines of the care and use of laboratory animals. The temperatures of the SS and DLC-Cu-SS electrosurgical devices were determined from thermographs obtained with a thermal-imaging infrared camera (Advanced Thermo TVS-500EX, NEC Avio Technologies, Tokyo, Japan). The highest temperature was recorded for every test.

Conventional SS and DLC-Cu-SS electrosurgical devices were used with an electrosurgical unit (ICC300H, EBRE, USA). The operation was performed under general anesthesia with isoflurane inhalation. After adequate skin preparation and sterilization, the liver was exposed through midline laparotomy, carefully avoiding liver injury. An electrosurgical device was inserted into the liver ( $2 \mathrm{~mm}$ deep) to create an anterior lesion, using a fixed power setting $(50 \mathrm{~W})$ and fixed activation time $(5 \mathrm{~s})$. The power setting and activation time are commonly used parameters in studies, and their values were based on those from a previous study [16]. At a special sacrificed time, the rats were injected with xylazine and tiletamine-zolazepam mixed with an equal volume of intraperitoneal injection, which was administered at a dose of $0.1 \mathrm{~mL}$ per $100 \mathrm{~g}$ of body weight. The abdominal cavity of the rats was exposed to facilitate tests. The left and central lobes of the liver were exposed, using the same instrument control
Wake force, current, depth, and length with varying electrical burn equipment. The cuts were made in different parts of the same lobe and at the same speed. Starting from the left side, liver samples were collected progressively towards the center. The same animals were used to obtain liver samples, each of $1 \mathrm{~cm}^{3}$, using different electrical equipment. The obtained liver samples were immediately fixed in $4 \%$ formalin. Dehydrated alcohol was used along with xylene to clean the samples, which were then embedded in paraffin. A slicer was used to cut vertical slices of the samples to demonstrate the shear direction.

2.3. Statistical Analysis. The data were expressed as averages with standard errors. The data were analyzed using analysis of variance (ANOVA). All statistical analyses were performed using SPSS version 12.0 (SPSS, Inc., Chicago, IL, USA). Values of $p<0.05$ were considered significant.

\section{Results}

Figure 1(b) presents a cross section of the DLC-Cu-SS thin film with a thickness of approximately $250 \mathrm{~nm}$. Moreover, the selected area electrical diffraction pattern (SAEDP) consisting of ring spots reveals a nanopolycrystalline structure in the thin film. The size and number of nano-Cu particles were proportional to the $\mathrm{Cu}$ content; hence, the production of the DLC-Cu-SS thin films varied with the ratio of $\mathrm{CH}_{4}$ and Ar. The amorphous-like phase that was transformed into a polycrystalline phase was induced by $\mathrm{Cu}$ doping/ion bombardment and radical reactions, as shown in Figure 1(c).

Figure 2 shows the temperature distributions of the electrosurgical devices from the DLC-Cu-SS and SS groups. The temperature of the direct contact electrode region is above $125^{\circ} \mathrm{C}$ in the control group, and the maximum temperature of the region in the DLC-Cu-SS group is approximately $110.84^{\circ} \mathrm{C}$. The temperatures decreased with increasing distance from the electrosurgical device, as shown in the crosssection images in Figure 3. Figure 4 shows the relationship between the highest temperatures of the liver and surgical time using different coated film thicknesses. The maximum temperature of the control group was $126.87^{\circ} \mathrm{C}$ and that of the DLC-Cu-SS coated group decreased by $12.63 \%$.

The SEM images of the cell morphology on the DLC-CuSS surfaces after culturing for $72 \mathrm{~h}$ are shown in Figure 5. For the MG-63 cell, the surface is almost completely covered by the cells. Moreover, the filopodia of the cells were not only attached to the nanostructured surface but also flattened with numerous filopodia. The well capability of cell differentiation and proliferation were investigated on the surface. Therefore, DLC is a nontoxic and biocompatible material.

Figure 6 shows the highest temperatures recorded. The average highest temperature was $148.74 \pm 14.28^{\circ} \mathrm{C}$ in the control group and $125.74 \pm 13.87^{\circ} \mathrm{C}$ in the DLC-Cu-SS group. A significant difference of the total injury area between the DLC-Cu-SS and SS groups was observed. The injury point of the SS group was significantly higher than that of the DLC$\mathrm{Cu}$-SS group on day 3 (Figure 7). The total injury sites for both groups reduced, but the injury area in the SS group was 


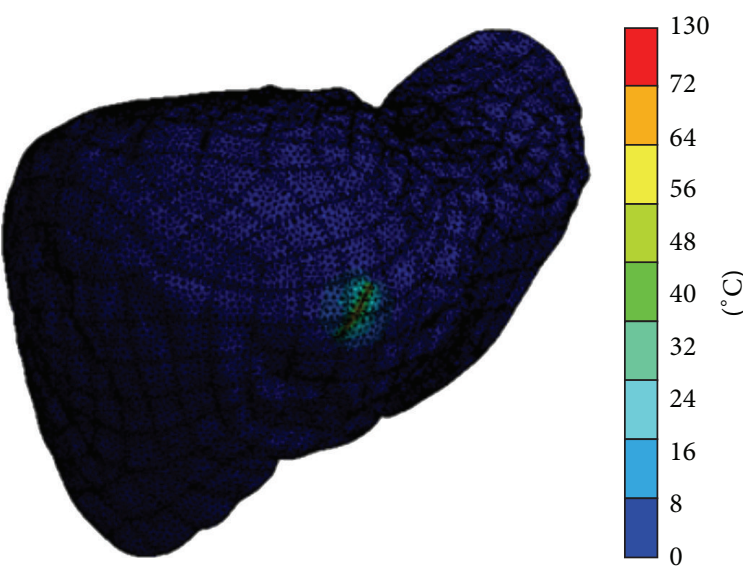

(a)

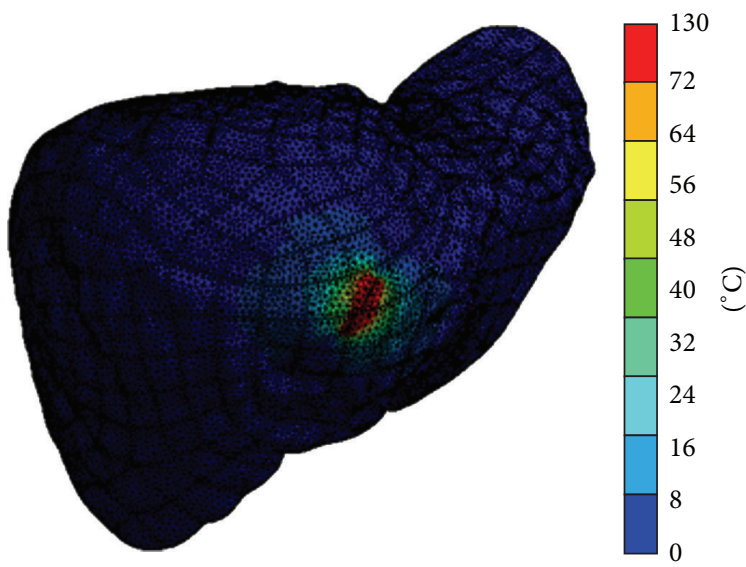

(c)

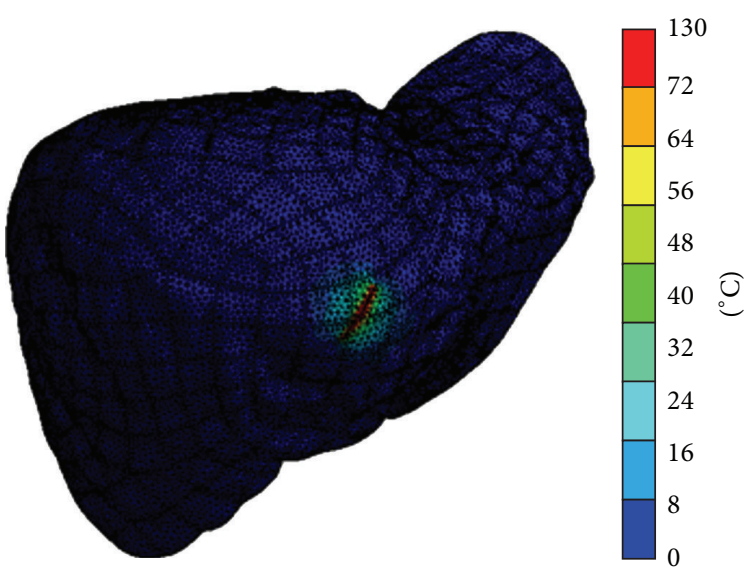

(e)

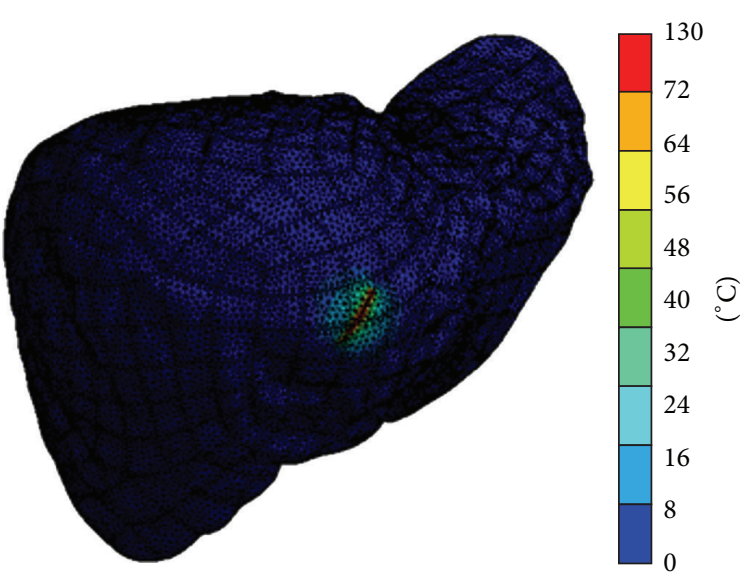

(b)

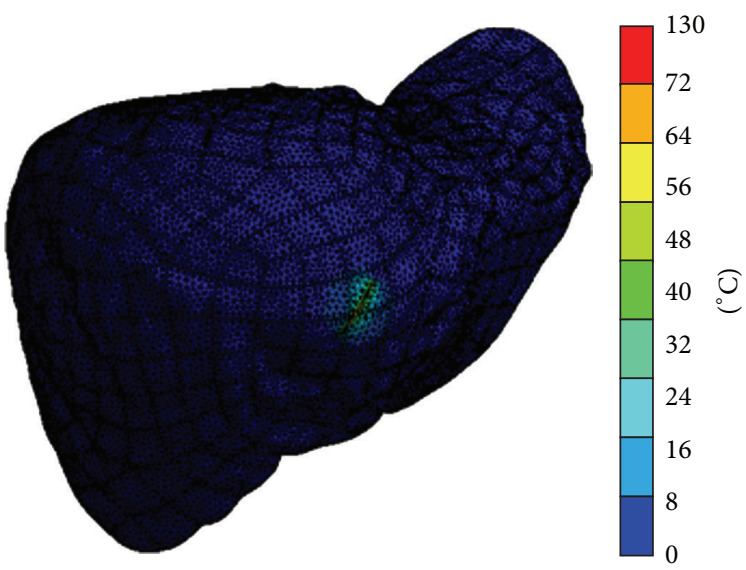

(d)

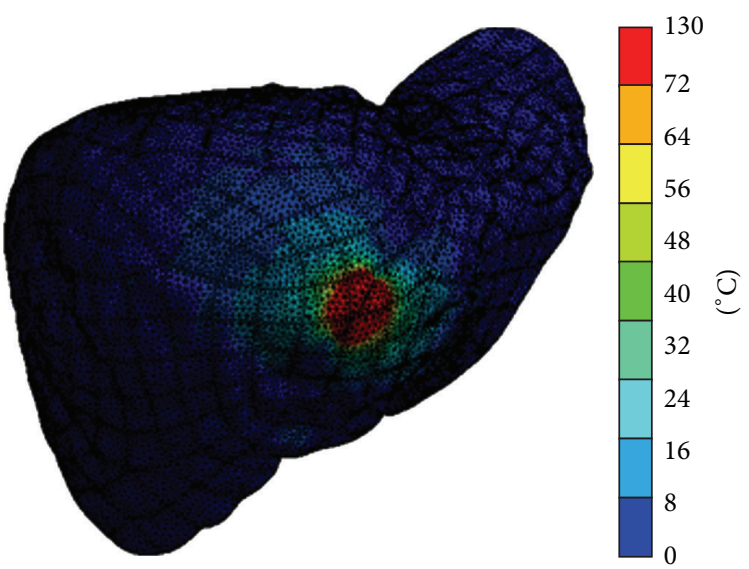

(f)

FIgURE 2: Temperature distributions of the liver in the DLC-Cu-SS-5 group at (a) 1, (b) 3, and (c) 5 seconds and in the SS group at (d) 1 , (e) 3 , and (f) 5 seconds.

still significantly larger than that in the DLC-Cu-SS group on day 28 (Figure 8 ). The average injury of tissue due to electrosurgery with an SS electrode $\left(3.41 \times 10^{3} \pm 147 \mathrm{~mm}^{2}\right)$ was significantly higher $(p<0.05)$ than that due to electrosurgery with a DLC-Cu-SS electrode $\left(2.74 \times 10^{3} \pm 119 \mathrm{~mm}^{2}\right)$ after 28 days. Fibrotic tissue appeared in both the SS and DLC-Cu-SS groups in the boundary between the thermalinjury region and the normal liver tissue on day 3. The nodular foci of hepatocytes were generated in the apoptotic area for both groups on day 28 . The SS group had a larger 


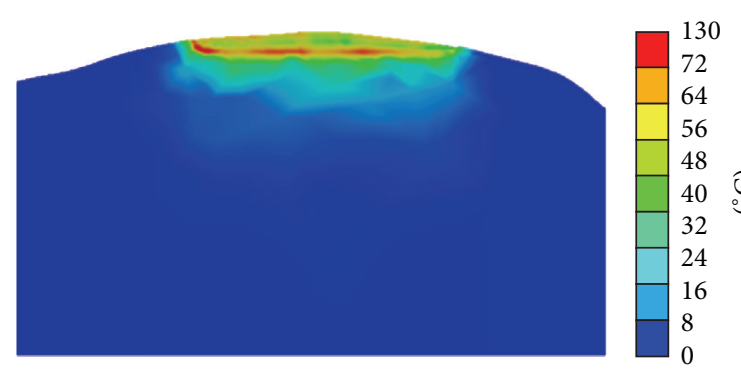

(a)

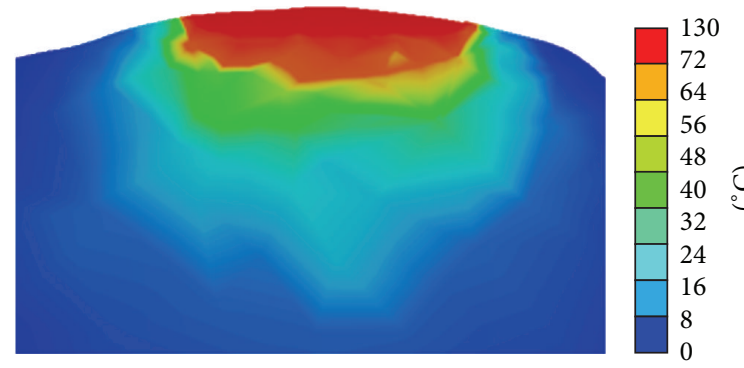

(c)

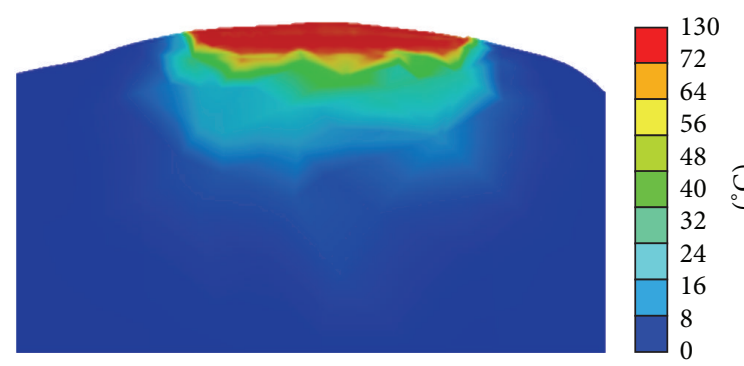

(e)

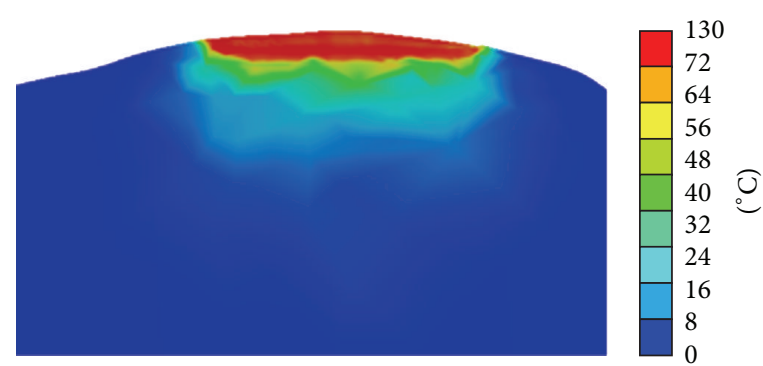

(b)

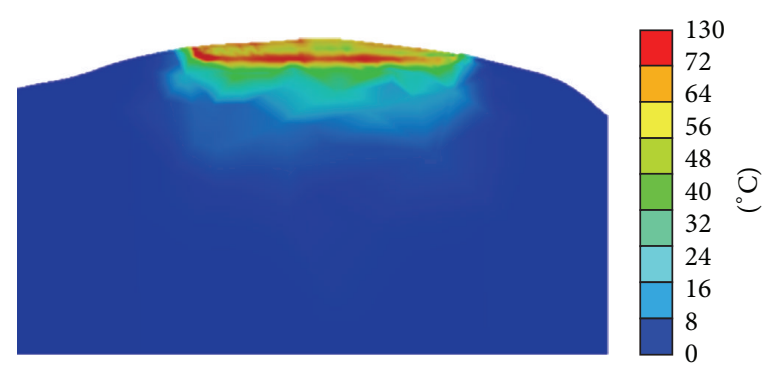

(d)

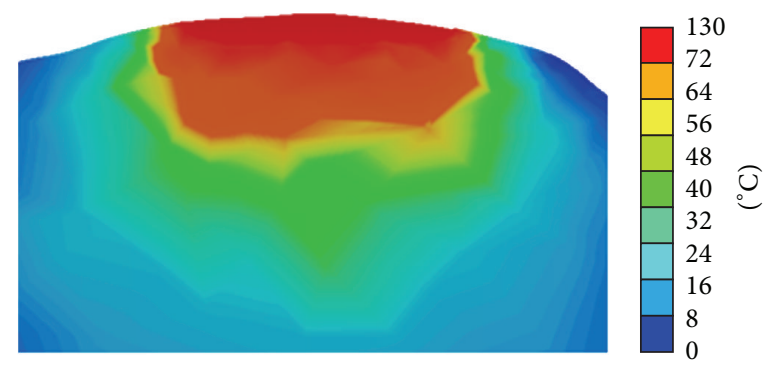

(f)

FIGURE 3: Temperature cross-section distributions of the liver in the DLC-Cu-SS-5 group at (a) 1, (b) 3, and (c) 5 seconds and in the SS group at (d) 1 , (e) 3 , and (f) 5 seconds.

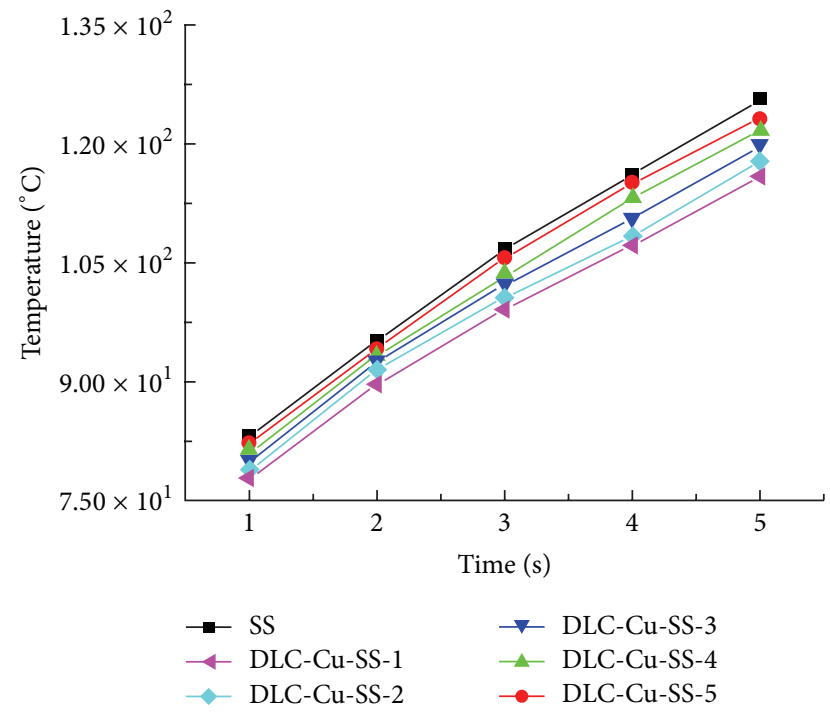

FIGURE 4: The highest temperatures in different thicknesses of DLC$\mathrm{Cu}$ film groups in the liver.

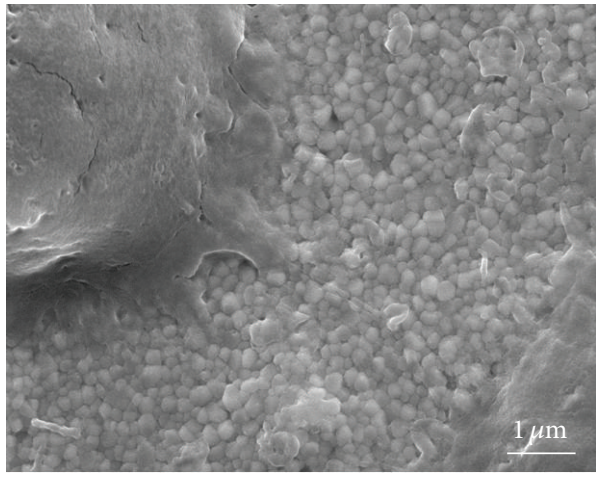

FIGURE 5: Cell morphology observation of the investigated samples SEM of MG-63 cell cultured on DLC-Cu surfaces for $72 \mathrm{~h}$.

fibrotic area than the DLC-Cu-SS group, and most apoptotic areas were replaced by regenerative hepatocyte foci in the DLC-Cu-SS group. 


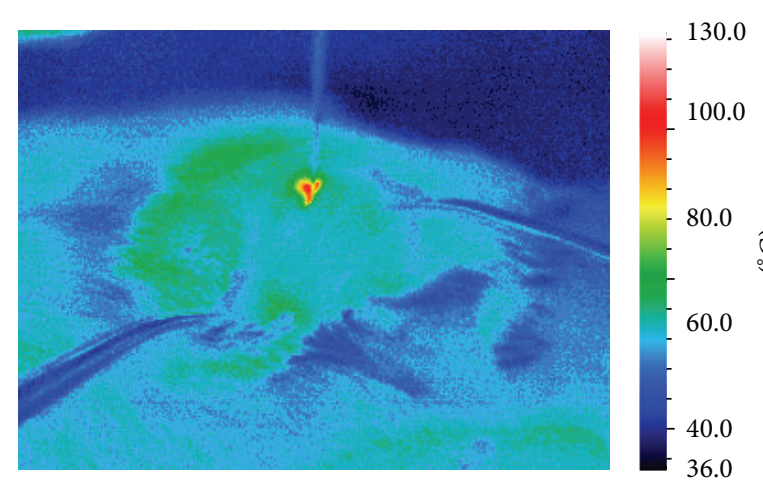

(a)

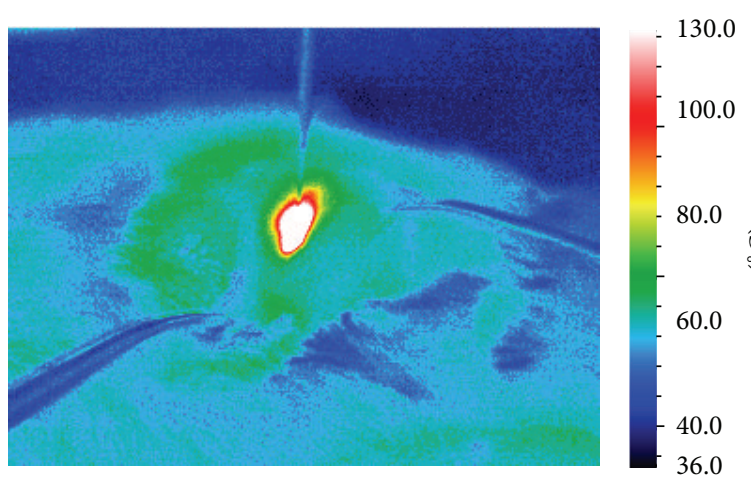

(c)
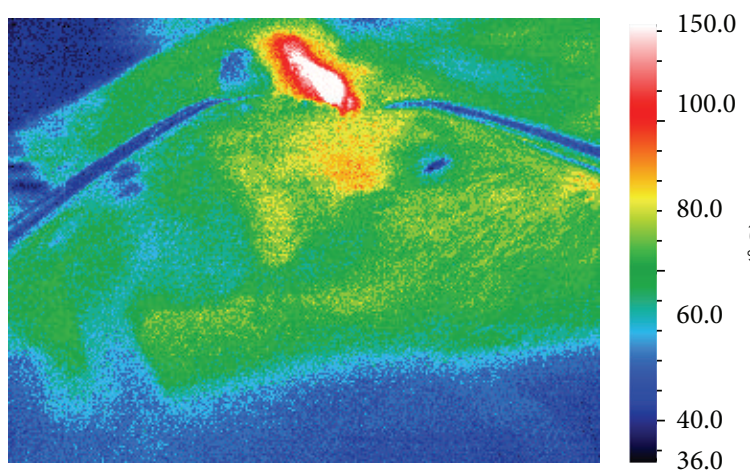

(e)

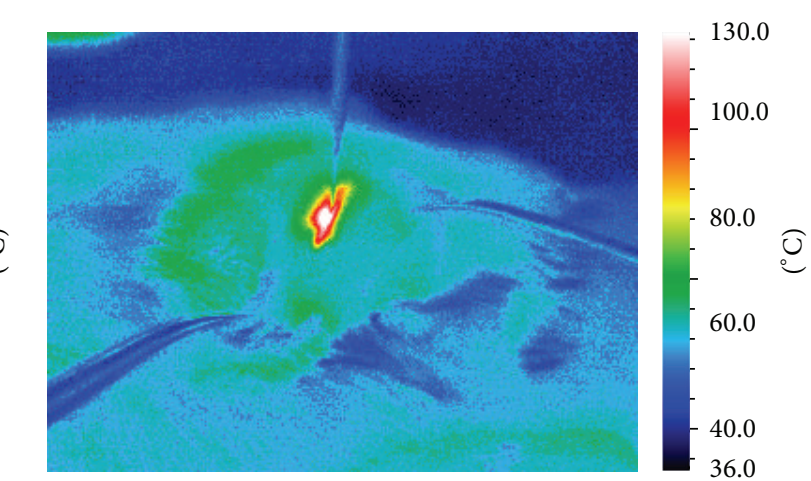

(b)

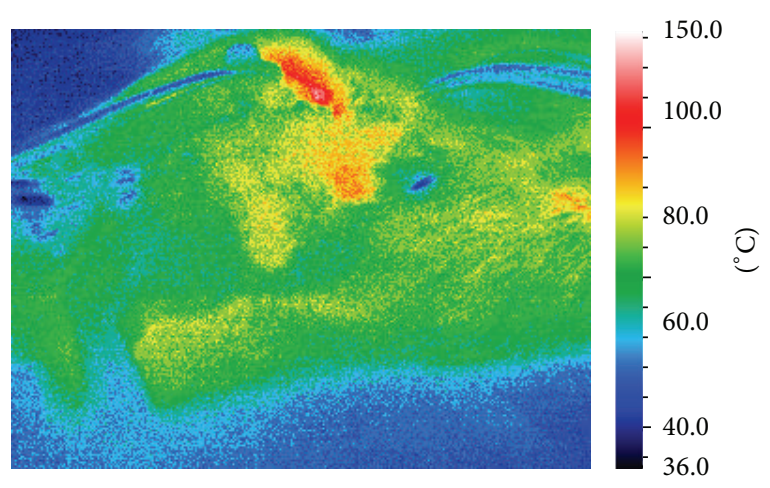

(d)

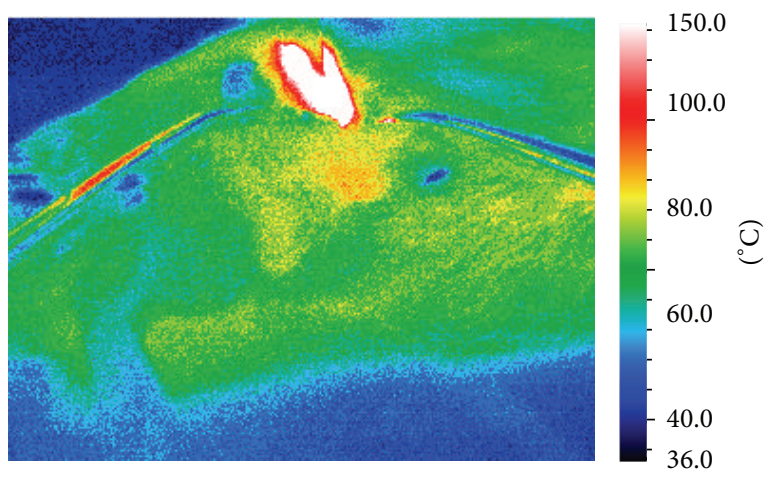

(f)

FIGURE 6: Highest recorded temperature for the DLC-Cu-SS-5 group at (a) 1, (b) 3, and (c) 5 seconds and in the SS group at (d) 1, (e) 3, and (f) 5 seconds.

\section{Discussion}

Despite the widespread use of electrosurgical devices in clinical surgeries, the surgical smoke, thermal injury, and tissue sticking caused by the devices are still issues that need to be addressed. Compared to complex irrigation or automatic thermal control, coating of an electrosurgical device with DLC-Cu-SS films to modify its surface properties is a relatively simple and economical approach to improve the instrument's performance. It is unnecessary to modify the design of the radio frequency generator or electrodes when a DLC-Cu-SS film is deposited on the electrode using thin film coating technology. Therefore, surface modification can be achieved in all types of electrosurgical devices.
Recently, the major problem in clinical surgery has been thermal damage to tissues. Solving the thermal-injury problem is challenging for designers. Remorgida developed instruments made of thermoinsulating plastic to create the electrode, and a metal conductor was incorporated in the plastic [17]. This instrument caused significantly less thermal damage to tissues. In addition, its lateral thermal diffusion is superior to that of standard bipolar forceps. Plastic can absorb the heat inside the jaws, so that coagulation occurs faster. By terminating the electrical current earlier, an overall reduction in temperature and tissue damage can be achieved.

If we apply electrosurgical device with surface treatment on the tissue with the nerve around, the thermal damage will hurt the nerve less. On the other hand, Carlander 


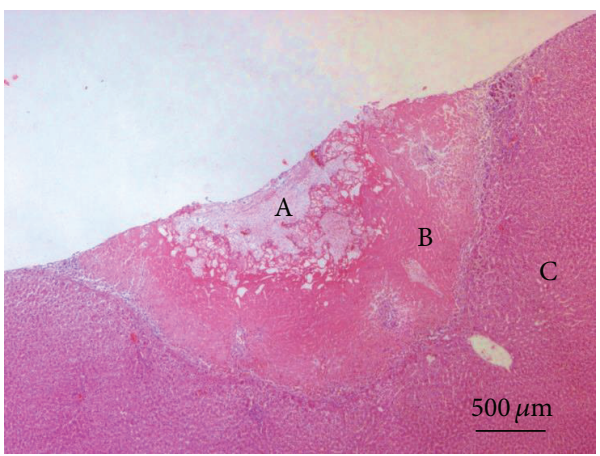

(a)

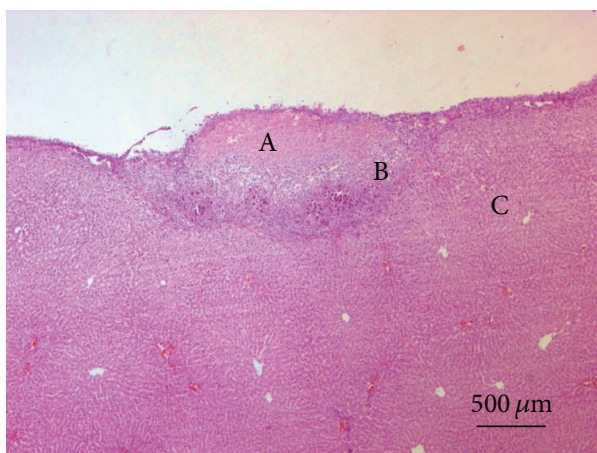

(c)

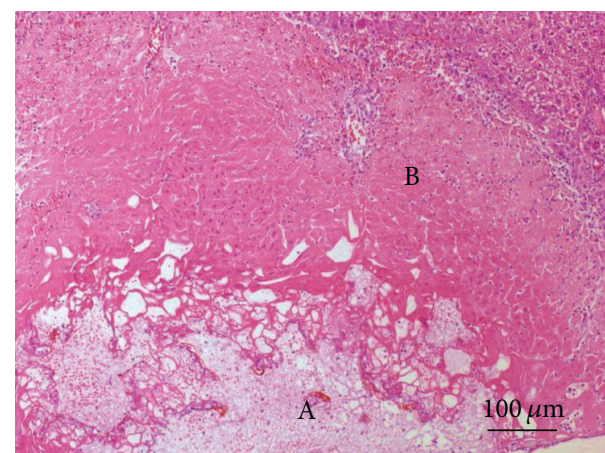

(b)

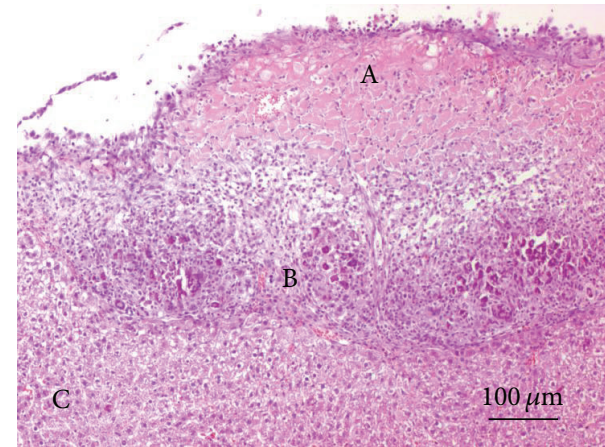

(d)

FIGURE 7: The total injury cross-section areas for the (a) DLC-Cu-SS group and (b) its amplification image and (c) control group and (d) its amplification image at day 3 (region A: injury area, region B: fibrotic tissue, and region C: normal tissue).

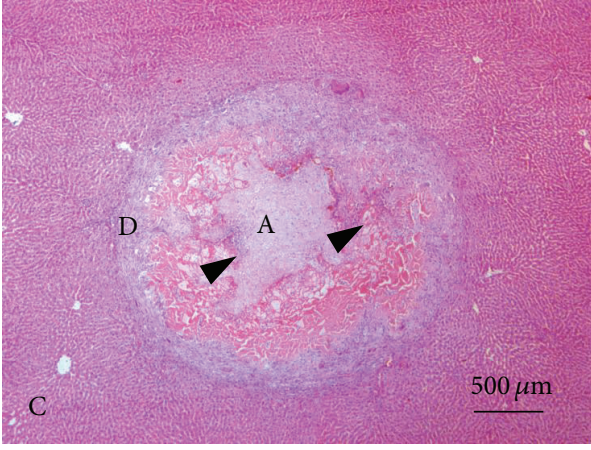

(a)

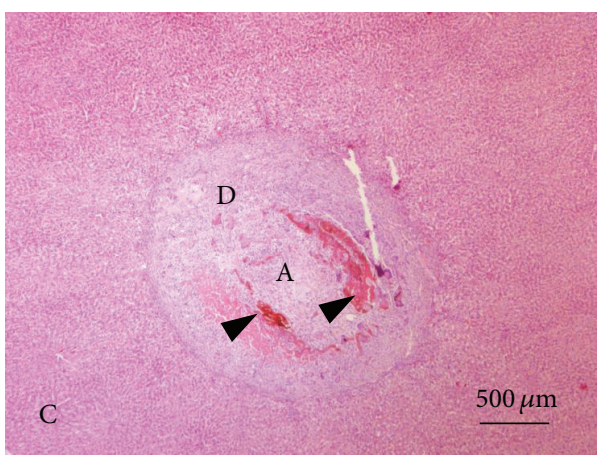

(c)

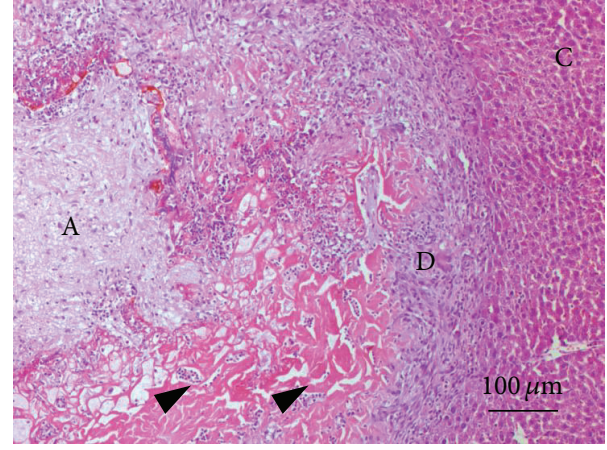

(b)

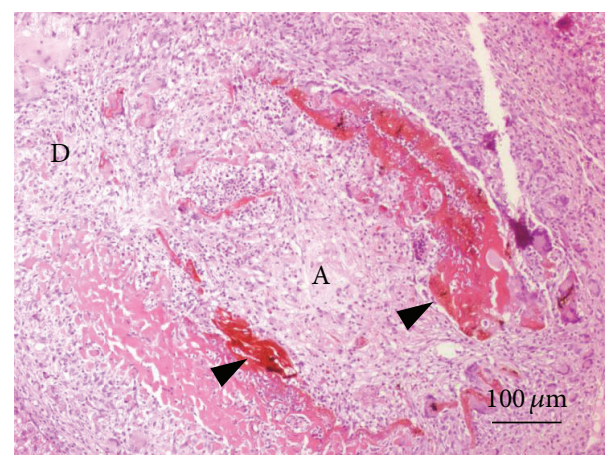

(d)

Figure 8: The total injury areas for the (a) DLC-Cu-SS group and (b) its amplification image and (c) control group and (d) its amplification image at day 28 (region A: injury area, region C: normal tissue, region D: apoptotic area, and arrow: nodular foci of hepatocytes). 
suggested adopting the ultrasonically activated scalpel (US) in the surgery surrounding nerve [18]. The desired effects of ultrasound on tissues can be achieved at temperatures up to $150^{\circ} \mathrm{C}$, whereas, in electrosurgery, the temperatures can reach as high as $400^{\circ} \mathrm{C}$. A higher lateral spread of energy was achieved with electrosurgical device, and bipolar electrosurgery resulted in tissue damage ten times greater than that with ultrasonic energy. With electrosurgery, the tissues appeared severely injured; coagulated fibers with myelin sheaths that were markedly thickened, homogeneous, and pale were observed. In contrast, with the US instruments, the tissue was only slightly injured; no apparent thickening of the myelin sheath and small myelin vacuoles were observed. Morphological examination showed significantly less nerve damage when US instruments were used. These instruments may be safer than electrosurgery for dissections that are close to nerves. Portella used pigs to operate the extrahepatic bile ducts during laparoscopic cholecystectomy, because pigs are medium-sized and easy to anesthetize; the pig's gall bladder and extrahepatic bile ducts are very similar to those of humans [19]. One of the pigs had a $1.2 \mathrm{~mm}$ long thermal injury of the cystic stump under an electrical power of $20 \mathrm{~W}$; hence, surface treatment is required for the electrosurgery to reduce the thermal damage.

Finite element models are used in thermal engineering analysis for decades. They have been successfully applied in biological applications to various human models [20]. Models have been used to determine liver tissue temperature in different biological structures. Most FEA studies analyze the biomechanical behavior of individual structures or materials. However, biomechanical models of the human finite element model are not perfect; on the whole, they are based on a number of assumptions and simplifications [21]. To obtain the correct geometry of the 3D liver model, the precise thickness $(1 \mathrm{~mm})$ for reconstruction was selected using MRI images. MRI can also be used to obtain information about the structure of liver tissues without exposing the patient to radiation. The contours of the hard tissue are not rigorously represented in MRI data. However, since our study focuses on the soft tissues of the liver, MRI was adopted.

It is difficult to accurately compare the results of this experiment with those obtained using models in previous studies, because of various differences in the material properties, muscle forces, mesh, and constrained conditions employed in the models. Another important factor that governs the analysis of the results is the mechanical and physical properties of the models relative to those of the actual object. The experiment conducted considered three main materials: liver tissue, SS, and DLC. The FEA study provided useful information on the temperature distribution in the liver. The limitations of our study are as follows: (1) a uniform surgical position was adopted; (2) vessels in the liver were not taken into account; (3) the model did not include other nearby organs; and (4) the study was only based on a theoretical analysis.

In animal studies, a typical injury-healing process is achieved through hemostasis, inflammation, proliferation, and remodeling [22]. The SS electrode resulted in a larger total injury area and thermal area than the DLC-Cu-SS electrode on day 3. On day 28 , in spite of some fibrotic tissues observed in the DLC-Cu-SS-electrode group, most of the apoptotic area was replaced by hepatocyte foci, which represented the late stage of liver remodeling [23, 24]. Although the remodeling processes were similar, the total injury area for the DLC-Cu-SS-electrode group was smaller than that of the SS-needle-electrode group. Overall, the animal study showed that the DLC-Cu-SS electrosurgical device caused a smaller injury area with a smaller thermal-injury site than the SS electrosurgical device. The smaller thermal-injury site resulted in less fibrotic tissue and a faster neogenesis process, which was beneficial to the liver-remodeling process.

\section{Conclusion}

The DLC-Cu film can be used to improve electrosurgery in terms of thermal injury and tissue remodeling using the surface treatment method. By using electrodes with surface treatment, the maximum temperature effectively decreases, the relative area of thermal injury and the fibrotic tissue decrease, and the speed of hepatic regeneration in the rat liver increases. DLC-Cu films also have other functions, such as enhancing hemocompatibility and antiadhesion capability $[25,26]$. As described above, DLC-Cu surface treatment in microsurgery applications can promote thermal conductivity, which is beneficial for clinical applications.

\section{Disclosure}

Ya-Ju Hsu is the first coauthor.

\section{Conflict of Interests}

The authors declare that they have no conflict of interests.

\section{Acknowledgments}

The authors would like to thank the Department of Health, Executive Yuan, Taiwan, under Contract no. MOHW103TDU-N-211-133001 and Health and welfare surcharge of tobacco products under Contract no. MOHW103-TD-B-11101 .

\section{References}

[1] S. Duffy, "3 The tissue and thermal effects of electrosurgery in the uterine cavity," Bailliere's Clinical Obstetrics and Gynaecology, vol. 9, no. 2, pp. 261-277, 1995.

[2] R. E. Fine and J. G. Vose, "Traditional electrosurgery and a low thermal injury dissection device yield different outcomes following bilateral skin-sparing mastectomy: a case report," Journal of Medical Case Reports, vol. 5, article 212, 2011.

[3] C. Koch, T. Friedrich, F. Metternich, A. Tannapfel, H.-P. Reimann, and U. Eichfeld, "Determination of temperature elevation in tissue during the application of the harmonic scalpel," Ultrasound in Medicine and Biology, vol. 29, no. 2, pp. 301-309, 2003. 
[4] H.-Y. Chen, C.-J. Tsai, and F.-H. Lu, "The Young's modulus of chromium nitride films," Surface and Coatings Technology, vol. 184, no. 1, pp. 69-73, 2004.

[5] T. Mikami, A. Takahashi, K. Hashi, S. Gasa, and K. Houkin, "Performance of bipolar forceps during coagulation and its dependence on the tip material: a quantitative experimental assay," Journal of Neurosurgery, vol. 100, no. 1, pp. 133-138, 2004.

[6] L. Zhang, P. Lv, Z. Y. Huang et al., "Blood compatibility of $\mathrm{La}_{2} \mathrm{O}_{3}$ doped diamond-like carbon films," Diamond and Related Materials, vol. 17, no. 11, pp. 1922-1926, 2008.

[7] Y.-H. Chan, C.-F. Huang, K.-L. Ou, and P.-W. Peng, "Mechanical properties and antibacterial activity of copper doped diamondlike carbon films," Surface and Coatings Technology, vol. 206, no. 6, pp. 1037-1040, 2011.

[8] E. A. Morra and A. S. Greenwald, "Polymer insert stress in total knee designs during high-flexion activities: a finite element study," The Journal of Bone and Joint Surgery-American Volume, vol. 87, supplement 2, pp. 120-124, 2005.

[9] V. K. Goel, M. M. Panjabi, A. G. Patwardhan, A. P. Dooris, and H. Serhan, "Test protocols for evaluation of spinal implants," The Journal of Bone and Joint Surgery-American Volume, vol. 88, supplement 2, pp. 103-109, 2006.

[10] R. A. Poggie, T. R. Turgeon, and R. D. Coutts, "Failure analysis of a ceramic bearing acetabular component," The Journal of Bone \& Joint Surgery Series A, vol. 89, no. 2, pp. 367-375, 2007.

[11] A. K. Ward, C. M. Ladtkow, and G. J. Collins, "Material removal mechanisms in monopolar electrosurgery," in Proceedings of the 29th Annual International Conference of IEEE-EMBS, Engineering in Medicine and Biology Society (EMBC '07), pp. 1180-1183, IEEE, Lyon, France, August 2007.

[12] J. J. Olaya, S. E. Rodil, S. Muhl, and E. Sánchez, "Comparative study of chromium nitride coatings deposited by unbalanced and balanced magnetron sputtering," Thin Solid Films, vol. 474, no. 1-2, pp. 119-126, 2005.

[13] I. dos Santos, D. Haemmerich, C. da Silva Pinheiro, and A. Ferreira da Rocha, "Effect of variable heat transfer coefficient on tissue temperature next to a large vessel during radiofrequency tumor ablation," BioMedical Engineering Online, vol. 7, article 21, 2008.

[14] N. Savvides and T. J. Bell, "Hardness and elastic modulus of diamond and diamond-like carbon films," Thin Solid Films, vol. 228, no. 1-2, pp. 289-292, 1993.

[15] H. M. Ledbetter, N. V. Frederick, and M. W. Austin, "Elasticconstant variability in stainless-steel 304," Journal of Applied Physics, vol. 51, no. 1, pp. 305-309, 1980.

[16] E. W. Elliott-Lewis, A. M. Mason, and D. L. Barrow, "Evaluation of a new bipolar coagulation forceps in a thermal damage assessment," Neurosurgery, vol. 65, no. 6, pp. 1182-1187, 2009.

[17] V. Remorgida, "Tissue thermal damage caused by bipolar forceps can be reduced with a combination of plastic and metal," Surgical Endoscopy, vol. 12, no. 7, pp. 936-939, 1998.

[18] J. Carlander, K. Johansson, S. Lindström, Å. K. Velin, C. H. Jiang, and C. Nordborg, "Comparison of experimental nerve injury caused by ultrasonically activated scalpel and electrosurgery," British Journal of Surgery, vol. 92, no. 6, pp. 772-777, 2005.

[19] A. O. Valoria Portella, M. R. MacIel Trindade, L. Z. Dias, S. Goldenberg, and E. N. Trindade, "Monopolar electrosurgery on the extrahepatic bile ducts during laparoscopic cholecystectomy: an experimental controlled trial," Surgical Laparoscopy, Endoscopy \& Percutaneous Techniques, vol. 19, no. 3, pp. 213-216, 2009.
[20] M. Sevimay, F. Turhan, M. A. Kiliçarslan, and G. Eskitascioglu, "Three-dimensional finite element analysis of the effect of different bone quality on stress distribution in an implantsupported crown," The Journal of Prosthetic Dentistry, vol. 93, no. 3, pp. 227-234, 2005.

[21] E. Tanaka, K. Tanne, and M. Sakuda, "A three-dimensional finite element model of the mandible including the TMJ and its application to stress analysis in the TMJ during clenching," Medical Engineering \& Physics, vol. 16, no. 4, pp. 316-322, 1994.

[22] S. Guo and L. A. Dipietro, "Factors affecting wound healing," Journal of Dental Research, vol. 89, no. 3, pp. 219-229, 2010.

[23] I. P. J. Alwayn, J. E. Verbesey, S. Kim et al., "A critical role for matrix metalloproteinases in liver regeneration," Journal of Surgical Research, vol. 145, no. 2, pp. 192-198, 2008.

[24] K. Moriya, E. Bae, K. Honda et al., "A fibronectin-independent mechanism of collagen fibrillogenesis in adult liver remodeling," Gastroenterology, vol. 140, no. 5, pp. 1653-1663, 2011.

[25] N. Huang, P. Yang, Y. X. Leng et al., "Surface modification of biomaterials by plasma immersion ion implantation," Surface and Coatings Technology, vol. 186, no. 1-2, pp. 218-226, 2004.

[26] P. Qi, M. F. Maitz, and N. Huang, "Surface modification of cardiovascular materials and implants," Surface and Coatings Technology, vol. 233, pp. 80-90, 2013. 

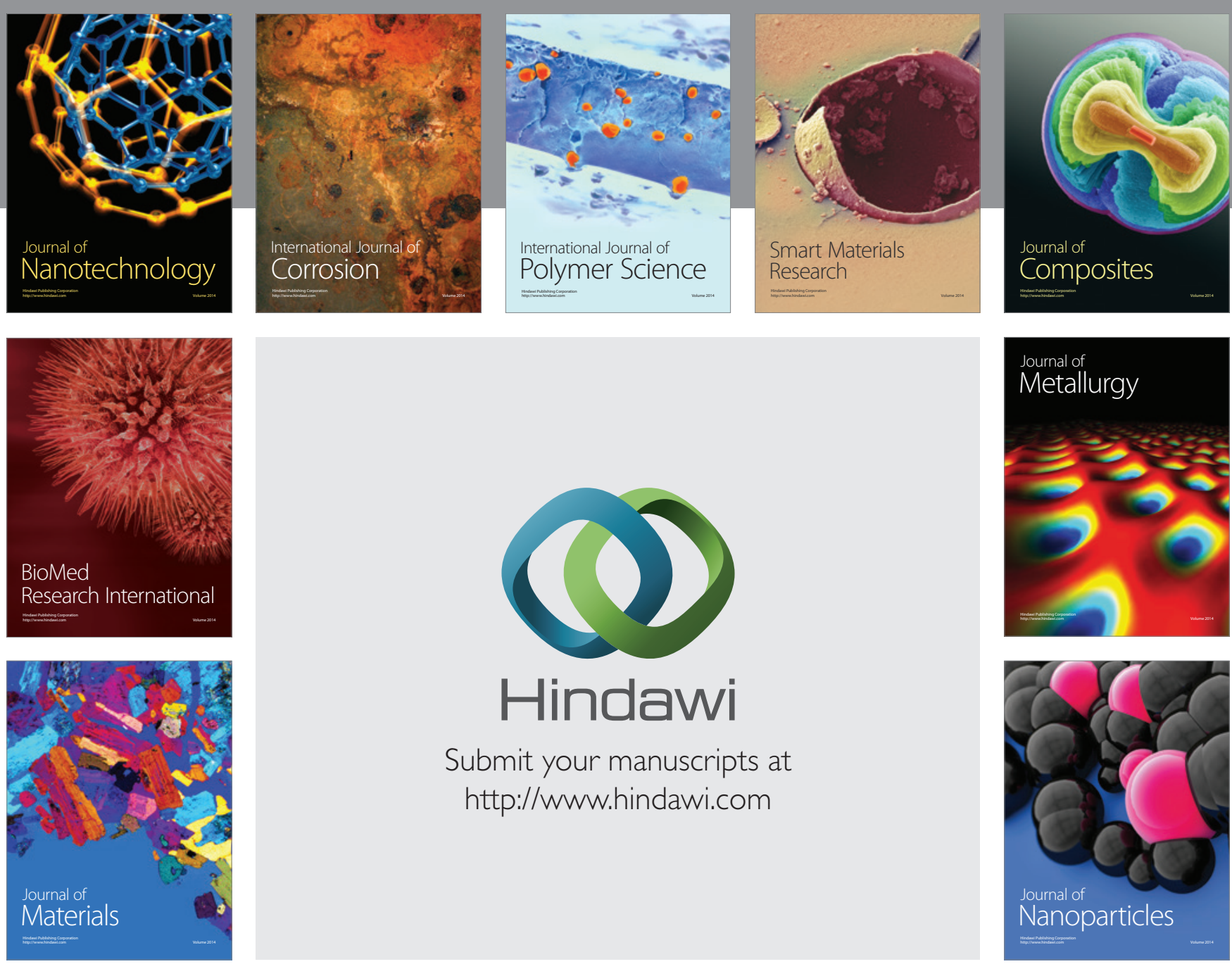

Submit your manuscripts at http://www.hindawi.com
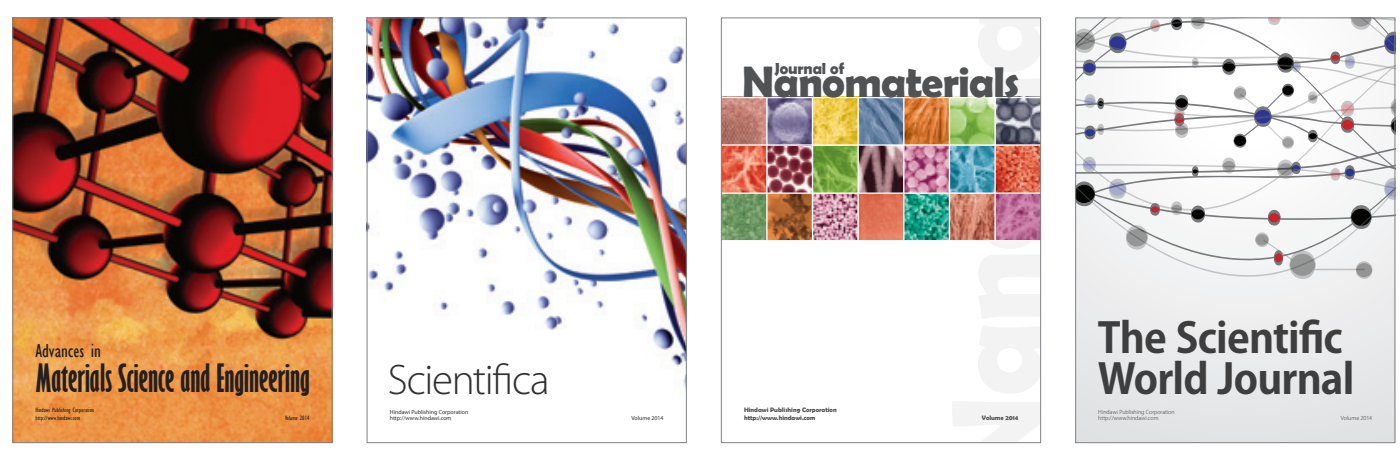

\section{The Scientific World Journal}
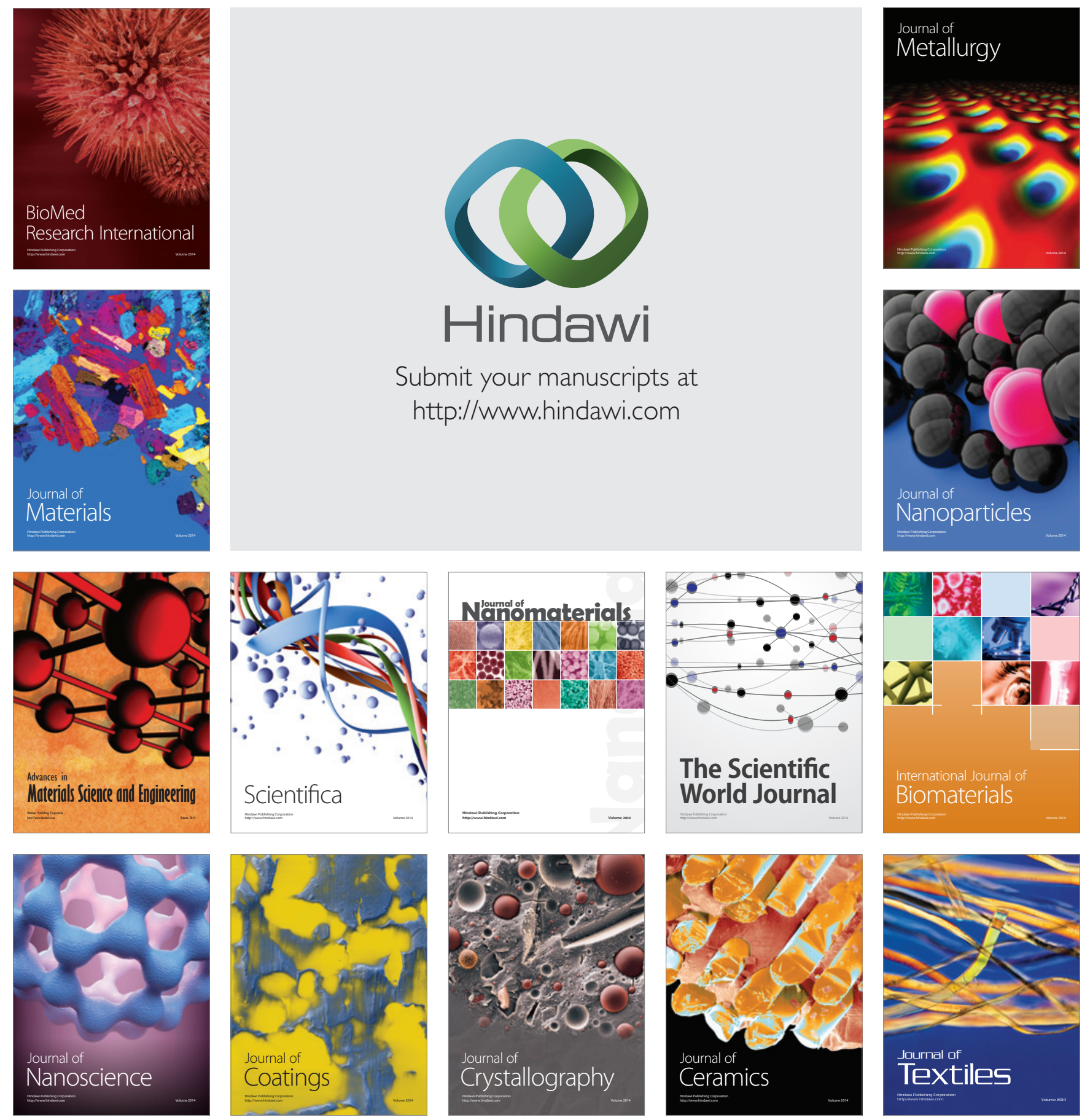